\title{
Prescribed Learning of R.E. Classes
}

\author{
Sanjay Jain ${ }^{\star 1}$, Frank Stephan*2 and Nan $\mathrm{Ye}^{3}$ \\ 1 Department of Computer Science, \\ National University of Singapore, Singapore 117543, Republic of Singapore. \\ Email sanjay@comp.nus.edu.sg \\ ${ }^{2}$ Department of Computer Science and Department of Mathematics, \\ National University of Singapore, Singapore 117543, Republic of Singapore. \\ Email fstephan@comp.nus.edu.sg \\ 3 Department of Computer Science and Department of Mathematics, \\ National University of Singapore, Singapore 117543, Republic of Singapore. \\ Email u0407028@nus.edu.sg
}

\begin{abstract}
This work extends studies of Angluin, Lange and Zeugmann on the dependence of learning on the hypotheses space chosen for the class. In subsequent investigations, uniformly recursively enumerable hypotheses spaces have been considered. In the present work, the following four types of learning are distinguished: class-comprising (where the learner can choose a uniformly recursively enumerable superclass as hypotheses space), class-preserving (where the learner has to choose a uniformly recursively enumerable hypotheses space of the same class), prescribed (where there must be a learner for every uniformly recursively enumerable hypotheses space of the same class) and uniform (like prescribed, but the learner has to be synthesized effectively from an index of the hypothesis space). While for explanatory learning, these four types of learnability coincide, some or all are different for other learning criteria. For example, for conservative learning, all four types are different. Several results are obtained for vacillatory and behaviourally correct learning; three of the four types can be separated, however the relation between prescribed and uniform learning remains open. It is also shown that every (not necessarily uniformly recursively enumerable) behaviourally correct learnable class has a prudent learner, that is, a learner using a hypotheses space such that it learns every set in the hypotheses space. Moreover the prudent learner can be effectively built from any learner for the class.
\end{abstract}

\section{Introduction}

The intuition behind learning in inductive inference [10] is that a learner sees more and more data and while reading the data produces conjectures about the concept to be learned which eventually stabilize on a correct description. The learning task is not arbitrary, but stems from a given class of concepts. Angluin [1] considered the important case that such a class is given

\footnotetext{
* Supported in part by NUS grant number R252-000-212-112 and R252-000-308-112.
} 
by an indexed family, that is, the class is uniformly recursive. She has given a characterization when such a class is explanatorily learnable and introduced also important variants such as conservative learning. In the present work, the more general case of uniformly r.e. classes is addressed. Previously learnability of uniformly r.e. classes had been considered by de Jongh, Kanazawa [7] and Zilles [26, 27].

Remark 1. First some basic notation. Let $W_{0}, W_{1}, W_{2}, \ldots$ be an acceptable enumeration of all r.e. subsets of the set of natural numbers $\mathbb{N}$. A language is a r.e. subset of natural numbers. Let $\varphi_{e}$ denote the $e$-th partial recursive function, again from an acceptable numbering. For more information on recursion theory, the reader is referred to standard text books like the ones of Odifreddi [18] and Soare [21]. The function $\langle e, x\rangle=\frac{1}{2} \cdot(e+x)(e+x+1)+x$ is Cantor's pairing function. A family $L_{0}, L_{1}, L_{2}, \ldots$ is uniformly recursively enumerable iff $\left\{\langle e, x\rangle: x \in L_{e}\right\}$ is a recursively enumerable set. For ease of notation, uniformly r.e. classes are just called r.e. classes. Note that in this paper, notations like $\left\{L_{0}, L_{1}, L_{2}, \ldots\right\}$ are used as a short-hand for both, the family as well as for the class of the sets; so set-theoretic comparisons like $\left\{L_{0}, L_{1}, L_{2}, \ldots\right\} \subseteq\left\{H_{0}\right.$, $\left.H_{1}, H_{2}, \ldots\right\}$ and $\left\{L_{0}, L_{1}, L_{2}, \ldots\right\}=\left\{H_{0}, H_{1}, H_{2}, \ldots\right\}$ ignore the ordering of the sets inside the class. Furthermore, let $W_{e, s}, L_{e, s}, H_{e, s}$ be the elements enumerated within time $s$ into $W_{e}, L_{e}, H_{e}$, respectively. Without loss of generality, $W_{e, s}, L_{e, s}, H_{e, s}$ are subsets of $\{0,1, \ldots, s\}$.

Let $\sigma, \tau$ range over $(\mathbb{N} \cup\{\#\})^{*}$. Furthermore, let $\sigma \subseteq \tau$ denote that $\tau$ is an extension of $\sigma$ as a string. content $(\sigma)$ denotes the set of natural numbers in the range of $\sigma$. T is a text if $T$ maps $\mathbb{N}$ to $\mathbb{N} \cup\{\#\}$ and $T$ is a text for $L_{a}$ iff the numbers occurring in $T$ are exactly those in $L_{a}$. content $(T)$ denotes the set of natural numbers in the range of $T . T[n]$ denotes the string consisting of the first $n$ elements of the text $T$, so $T[0]$ is the empty string and $T[2]=T(0) T(1)$.

Remark 2. A learner is a recursive function from $(\mathbb{N} \cup\{\#\})^{*}$ to $\mathbb{N} \cup\{$ ? $\}$. In the following, let $M$ be a learner and let $\left\{L_{0}, L_{1}, L_{2}, \ldots\right\},\left\{H_{0}, H_{1}, H_{2}, \ldots\right\}$ be r.e. classes. Here $\left\{L_{0}, L_{1}, L_{2}, \ldots\right\}$ is the class $M$ should learn and $\left\{H_{0}, H_{1}, H_{2}, \ldots\right\}$ is the hypotheses space used by $M$.

The learner $M$ converges on $T$ to $b$ if there is an $n$ with $M(T[m])=b$ for all $m \geq n$.

The learner $M$ is finite [10] if for every text $T$ there is one index $e$ such that for all $n$, either $M(T[n])=$ ? or $M(T[n])=e$.

The learner $M$ is confident [19] if $M$ converges on every text $T$ to a hypothesis.

The learner $M$ is conservative [1] if for all $\sigma, \tau$ with $M(\sigma \tau) \neq M(\sigma)$ there is an $x$ occurring in $\sigma \tau$ such that $x \notin H_{M(\sigma)}$.

The learner $M$ semantically identifies $L_{a}$ if, given any text $T$ for $L, H_{M(T[n])}=L_{a}$ for almost all $n$. The learner $M$ syntactically identifies $L_{a}$ if, given any text $T$ for $L$, there is a $b$ with $H_{b}=L_{a}$ and $M(T[n])=b$ for almost all $n$.

The learner $M$ is a behaviourally correct learner for $\left\{L_{0}, L_{1}, L_{2}, \ldots\right\}$ iff $M$ semantically identifies every $L_{a}[3,6] ; M$ is an explanatory learner for $\left\{L_{0}, L_{1}, L_{2}, \ldots\right\}$ if $M$ syntactically identifies every $L_{a}[4,10] . M$ is a vacillatory learner for $\left\{L_{0}, L_{1}, L_{2}, \ldots\right\}$ iff $M$ is a behaviourally correct learner for $\left\{L_{0}, L_{1}, L_{2}, \ldots\right\}$ which on every text for a language $L_{a}$ outputs only finitely many syntactically different hypotheses [5].

The learner $M$ is prudent $[9,19]$ if it learns all languages in its hypotheses space $\left\{H_{0}, H_{1}\right.$, $\left.H_{2}, \ldots\right\}$. 
In the first three sections, all classes considered are recursively enumerable, only in Section 4 learnability of general classes is investigated.

Remark 3. Let $M$ be a learner for $\left\{L_{0}, L_{1}, L_{2}, \ldots\right\}$ using hypotheses space $\left\{H_{0}, H_{1}, H_{2}, \ldots\right\}$. A sequence $\sigma$ is called syntactic stabilizing sequence for $M$ on a set $L$ iff $\sigma \in(L \cup\{\#\})^{*}$ and for all $\tau \in(L \cup\{\#\})^{*}, M(\sigma \tau)=M(\sigma)$. A sequence $\sigma$ is called semantic stabilizing sequence for $M$ on a set $L$ iff $\sigma \in(L \cup\{\#\})^{*}$ and for all $\tau \in(L \cup\{\#\})^{*}, H_{M(\sigma \tau)}=H_{M(\sigma)}$. Stabilizing sequences are called locking sequences for $M$ on $L$, if in addition to the above conditions it holds that $H_{M(\sigma)}=L$. Note that, if $M$ learns $L$ then stabilizing sequences for $M$ on $L$ are also locking sequences for $M$ on $L$.

Let $K$ denote the halting problem. Let $K^{\prime}$ denote the halting problem relative to $K$. There is a partial $K$-recursive function $\Gamma$ which assigns to each $e$ the length-lexicographically least syntactic stabilizing sequence for $M$ on $L_{e} ; \Gamma(e)$ is defined iff such a sequence exists. $\Gamma$ has a twoplace approximation $\gamma(e, t)$ which converges to $\Gamma(e)$ if $\Gamma(e)$ is defined and diverges otherwise. Note that $\Gamma$ and $\gamma$ can be obtained effectively from an index for $M$ and an index $e^{\prime}$ with $W_{e^{\prime}}=\left\{\langle e, x\rangle: x \in L_{e}\right\}$. Blum and Blum [4] introduced the notion of locking sequences and Fulk [9] introduced the notion of stabilizing sequences.

Angluin [1], Lange, Kapur and Zeugmann [15, 16, 23-25] studied the dependence between the family $\left\{L_{0}, L_{1}, L_{2}, \ldots\right\}$ to be learned and the hypotheses space $\left\{H_{0}, H_{1}, H_{2}, \ldots\right\}$ used by the learner. To formalize this, they introduced the notions of exact, class-preserving and classcomprising learning. In addition to this, new notions like uniform and prescribed are introduced. Here $I$ ranges over properties of the learner as defined in Remark 2, so $I$ stands for "finite", "explanatory", "conservatively explanatory", "confidently explanatory", "vacillatory" and "behaviourally correct".

Definition 4. $\left\{L_{0}, L_{1}, L_{2}, \ldots\right\}$ is class-comprisingly $I$ learnable iff it is $I$ learnable with respect to some hypotheses space $\left\{H_{0}, H_{1}, H_{2}, \ldots\right\}$; note that learnability automatically implies $\left\{L_{0}, L_{1}\right.$, $\left.L_{2}, \ldots\right\} \subseteq\left\{H_{0}, H_{1}, H_{2}, \ldots\right\}$.

$\left\{L_{0}, L_{1}, L_{2}, \ldots\right\}$ is class-preservingly $I$ learnable iff it is $I$ learnable with respect to some hypotheses space $\left\{H_{0}, H_{1}, H_{2}, \ldots\right\}$ satisfying $\left\{H_{0}, H_{1}, H_{2}, \ldots\right\}=\left\{L_{0}, L_{1}, L_{2}, \ldots\right\}$.

$\left\{L_{0}, L_{1}, L_{2}, \ldots\right\}$ is prescribed $I$ learnable iff it is $I$ learnable with respect to every hypotheses space $\left\{H_{0}, H_{1}, H_{2}, \ldots\right\}$ such that $\left\{L_{0}, L_{1}, L_{2}, \ldots\right\}=\left\{H_{0}, H_{1}, H_{2}, \ldots\right\}$.

$\left\{L_{0}, L_{1}, L_{2}, \ldots\right\}$ is uniformly $I$ learnable iff there is a recursive enumeration of partialrecursive functions $M_{0}, M_{1}, M_{2}, \ldots$ such that the following holds: Whenever $\left\{H_{0}, H_{1}, H_{2}, \ldots\right\}=$ $\left\{L_{0}, L_{1}, L_{2}, \ldots\right\}$ and $W_{e}=\left\{\langle d, x\rangle: x \in H_{d}\right\}$ then $M_{e}$ is total and an $I$ learner for $\left\{L_{0}, L_{1}\right.$, $\left.L_{2}, \ldots\right\}$ with respect to this hypotheses space $\left\{H_{0}, H_{1}, H_{2}, \ldots\right\}$.

Remark 5. Lange and Zeugmann [15,23] considered besides class-preserving and class-comprising also the following notion: $\left\{L_{0}, L_{1}, L_{2}, \ldots\right\}$ is exactly $I$ learnable iff it is $I$ learnable with $\left\{L_{0}\right.$, $\left.L_{1}, L_{2}, \ldots\right\}$ itself taken as hypotheses space. Note that this notion needs that the ordering of the languages in $\left\{L_{0}, L_{1}, L_{2}, \ldots\right\}$ is taken into account, while all other definitions hold without paying attention to the specific ordering of the sets inside $\left\{L_{0}, L_{1}, L_{2}, \ldots\right\}$. The relation to 
prescribed learning is that a class $\left\{L_{0}, L_{1}, L_{2}, \ldots\right\}$ is prescribed $I$ learnable iff every family $\left\{H_{0}\right.$, $\left.H_{1}, H_{2}, \ldots\right\}$ with $\left\{H_{0}, H_{1}, H_{2}, \ldots\right\}=\left\{L_{0}, L_{1}, L_{2}, \ldots\right\}$ is exactly $I$ learnable.

The question whether a class can be learned using any given representation is quite natural. It reflects the situation where a company building learners cannot enforce its representation of the data/hypothesis on the clients but has to make for each client a learning algorithm using the client's representation. The difference between prescribed and uniform learning would then be that in the first case the programmers have to adjust for each client the learning program by hand, while in the second case there is some synthesizer which reads the clients requirements from some file and then adapts the learner automatically.

Remark 6. Note that in the case of learning with respect to r.e. families, uniform learning and prescribed learning are defined in a class-preserving way. Jain and Stephan [13] showed that there is a one-one numbering of all r.e. sets (that is a Friedberg Numbering [8]) such that only classes with finitely many infinite sets can be behaviourally correct learned with respect to this numbering as hypotheses space.

Furthermore, above result can be strengthened to uniform learning by showing that only classes consisting of finite sets are class-comprising-uniformly behaviourally correct learnable. To see this, let $\left\{H_{0}, H_{1}, H_{2}, \ldots\right\}$ be a Friedberg numbering [8]. For a given parameter $e$, a family $\left\{G_{0}\right.$, $\left.G_{1}, G_{2}, \ldots\right\}$ is constructed from $\left\{H_{0}, H_{1}, H_{2}, \ldots\right\}$ such that the following holds for all $a$ :

- For all $b, G_{\langle a, b\rangle} \subseteq H_{a}$;

$-G_{\langle a, b\rangle}=H_{a}$ if either $b=0 \wedge\left|W_{e}\right|=\infty$ or $b=\left|W_{e}\right|+1$;

$-G_{\langle a, b\rangle}$ is finite if either $b>0 \wedge\left|W_{e}\right|=\infty$ or $b \neq\left|W_{e}\right|+1 \wedge\left|W_{e}\right|<\infty$.

Suppose by way of contradiction that there is an r.e. infinite set $H_{a}$ such that some class containing $H_{a}$ can be class-comprising-uniformly behaviourally correctly learned. Note that for any fixed $e$ and the class $\left\{G_{0}, G_{1}, G_{2}, \ldots\right\}$ with parameter $e$ built as above, there exists exactly one index $\langle f(e), g(e)\rangle$ with $G_{\langle f(e), g(e)\rangle}=H_{a}$. By construction, $f(e)=a$. By the assumption on uniform learnability, there is a recursive enumeration of learners $N_{0}, N_{1}, N_{2}, \ldots$ such that each $N_{e}$ learns the given class with respect to the hypotheses space $\left\{G_{0}, G_{1}, G_{2}, \ldots\right\}$ built with parameter $e$. As there is a fixed recursive text $T$ for $H_{a}$ and one can simulate $N_{e}$ on $T$, the function $g$ is limit-recursive (that is, there exists a recursive function $h$ such that $g(x)=\lim _{t \rightarrow \infty} h(x, t)$ ). Note that $W_{e}$ is infinite iff $g(e)=0$. As $\left\{e:\left|W_{e}\right|=\infty\right\} \mathbb{Z}_{T} K$, this gives a contradiction. So class-comprising uniform behaviourally correct learning only permits to learn classes of finite sets.

Thus it is reasonable to restrict oneself to the class-preserving versions of prescribed and uniform learning; this convention has already been adapted in Definition 4.

The next result is obvious from the definitions.

Proposition 7. For any notion I of learning and any class $\mathcal{L}$, the following implications hold: $\mathcal{L}$ is uniformly I-learnable $\Rightarrow\left\{L_{0}, L_{1}, L_{2}, \ldots\right\}$ is prescribed I-learnable $\Rightarrow \mathcal{L}$ is class-preservingly I-learnable $\Rightarrow \mathcal{L}$ is class-comprisingly I-learnable. 
It depends on the chosen learning criterion $I$, which of the implications can be reversed. For finite and explanatory learning, all four notions are the same, as shown in Theorems 8 and 9. A lot of research [11] deals with requiring additional constraints on how hypotheses are chosen during explanatory learning. Such requirements change also the relations between the four types of learning. For confident learning, Theorem 10 shows that the uniform, prescribed and class-preserving type coincide while class-comprising confident learning is more general. For conservative learning, Example 11 gives classes which separate all four types of conservative learning. Theorems 12, 13, 15 and 16 deal with vacillatory and behaviourally correct learning. They give classes which, for these criteria, are class-comprisingly but not class-preservingly learnable as well as classes which are class-preservingly but not prescribed learnable. The separation of prescribed from uniform is open for these two criteria.

The importance of prudence is that the hypotheses space and the class of learned sets coincide; so the learner never conjectures some set it cannot learn. Fulk [9] showed that prudence is not restrictive for explanatory learning. Jain and Sharma [12] showed that prudence is not restrictive for vacillatory learning. In Theorem 17 it is shown that prudence is not restrictive for behaviourally correct learning. In 1988, Kurtz and Royer [14] had claimed to have this result, but their proof had a bug and the problem had remained open since then. Furthermore, the construction of the prudent learner is effective in the original learner for a behaviourally learnable class. It is still open whether prudence for explanatory and vacillatory learning can be effectivized.

\section{$2 \quad$ Finite and Explanatory Learning}

Finite learnable classes can be learnt uniformly, because finite learning is determined by a finite subset of the target language.

Theorem 8. Every class-comprisingly finitely learnable class is also uniformly finitely learnable.

Proof. Let $M$ be a finite learner for $\left\{L_{0}, L_{1}, L_{2}, \ldots\right\}$ using a class-comprising hypotheses space. Let $e$ be an index for a hypothesis space $\left\{H_{0}, H_{1}, H_{2}, \ldots\right\}$. That is, $W_{e}=\left\{\langle b, x\rangle: x \in H_{b}\right\}$. Further suppose $\left\{H_{0}, H_{1}, H_{2}, \ldots\right\}=\left\{L_{0}, L_{1}, L_{2}, \ldots\right\}$. Then a learner $M_{e}$ is defined as follows. $M_{e}(T[n])$ is defined by the first case below which applies:

- If there is an $m<n$ with $M_{e}(T[m]) \neq$ ? then $M_{e}(T[n])=M_{e}(T[m])$ for the least such $m$;

- If there are $m \leq n$ and $b \leq n$ with $M(T[m]) \neq ?$ and content $(T[m]) \subseteq H_{b, n}$ then $M_{e}(T[n])=b$;

- Otherwise $M_{e}(T[n])=$ ?.

The first condition guarantees that $M_{e}$ outputs on $T$ at most one hypothesis besides the symbol ?. Hence every $M_{e}$ is a finite learner. It follows from the definition of finite learning that $H_{b}=H_{c}$ whenever $M(T[m]) \neq$ ?, content $(T[m]) \subseteq H_{b}$ and content $(T[m]) \subseteq H_{c}$. Hence the $b$ chosen in the second case is a correct hypothesis whenever this case applies. Furthermore, this case eventually applies on texts for languages in $\left\{L_{0}, L_{1}, L_{2}, \ldots\right\}$. This completes the proof that $\left\{L_{0}\right.$, $\left.L_{1}, L_{2}, \ldots\right\}$ is uniformly finitely learnable.

The same result holds for explanatory learning. 
Theorem 9. Every class-comprisingly explanatorily learnable class is also uniformly explanatorily learnable.

Proof. Let $\mathcal{L}$ be given and let $M$ be a learner using a hypotheses space $\left\{L_{0}, L_{1}, L_{2}, \ldots\right\}$ containing $\mathcal{L}$ and perhaps other languages. Choose $i$ such that $W_{i}=\left\{\langle a, x\rangle: x \in L_{a}\right\}$.

Fix any $j$ and assume that $j$ is an index of a hypotheses space $\left\{H_{0}, H_{1}, H_{2}, \ldots\right\}$ for $\mathcal{L}$, that is, assume $\left\{H_{0}, H_{1}, H_{2}, \ldots\right\}=\mathcal{L}$ and $W_{j}=\left\{\langle b, x\rangle: x \in H_{b}\right\}$. Let $\Gamma_{j}$ be the function from Remark 3 which assigns to the members of $\left\{H_{0}, H_{1}, H_{2}, \ldots\right\}$ the length-lexicographically least syntactic stabilizing sequences with respect to the learner $M . \gamma_{j}(b, t)$ is then the $t$-th approximation of $\Gamma_{j}(b)$ as defined in Remark 3.

The learner $M_{j}$ is constructed as follows: $M_{j}(\sigma)$ is the least $b$ such that either $\gamma_{i}(M(\sigma),|\sigma|)$ $=\gamma_{j}(b,|\sigma|)$ or $b=|\sigma|$. The latter condition is just to make $M_{j}$ total and to terminate the search.

Assume that $M$ converges on some text $T$ to an index $a$ of a language $L_{a} \in \mathcal{L}$. As $L_{a} \in \mathcal{L}$, there is a $b$ with $H_{b}=L_{a}$; assume that $b$ is the least such index. As $\left\{H_{0}, H_{1}, H_{2}, \ldots\right\}=\mathcal{L}$ and $M$ is a learner for $\left\{H_{0}, H_{1}, H_{2}, \ldots\right\}$, an index $c$ satisfies $\Gamma_{j}(c)=\Gamma_{i}(a)$ iff $H_{c}=L_{a}$. Hence $M_{j}$ converges on $T$ to $b$ as, for all $c<b$ and almost all $s, \gamma_{j}(b, s)=\gamma_{i}(a, s)$ and $\gamma_{j}(c, s) \neq \gamma_{i}(a, s)$. It follows that $M_{j}$ learns $\mathcal{L}$ using the hypotheses space $\left\{H_{0}, H_{1}, H_{2}, \ldots\right\}$.

The next result shows that class-preserving confident learning coincides with uniform confident learning. The proof of the second part shows that class-preserving confident learning is not closed under taking subclasses.

Theorem 10. (a) Every class-preservingly confidently learnable class $\mathcal{L}$ is also uniformly confidently learnable.

(b) The class $\left\{D:|D|=2 \vee\left(|D|=1 \wedge D \subseteq K^{\prime}\right)\right\}$ is class-comprisingly but not classpreservingly confidently learnable.

Proof. (a) Reviewing the proof of Theorem 9, the additional constraints to those given there on $M$ and $\left\{L_{0}, L_{1}, L_{2}, \ldots\right\}$ are that $\left\{L_{0}, L_{1}, L_{2}, \ldots\right\}=\mathcal{L}$ and $M$ converges on every text to some index. Assume again that $j$ and $\left\{H_{0}, H_{1}, H_{2}, \ldots\right\}$ satisfy $\left\{L_{0}, L_{1}, L_{2}, \ldots\right\}=\left\{H_{0}, H_{1}, H_{2}, \ldots\right\}$ and $W_{j}=\left\{\langle b, x\rangle: x \in H_{b}\right\}$. Assume that $T$ is any text. Then $M$ converges on $T$ to some index $a$ as $M$ is confident. By construction, $M_{j}$ converges then to the least index $b$ with $L_{a}=H_{b}$. Hence $M_{j}$ also converges on all texts and hence $M_{j}$ is confident. Furthermore, $M_{j}$ learns $\mathcal{L}$ explanatorily with respect to the hypotheses space $\left\{H_{0}, H_{1}, H_{2}, \ldots\right\}$.

(b) The class $\left\{D:|D|=2 \vee\left(|D|=1 \wedge D \subseteq K^{\prime}\right)\right\}$ is class-comprisingly confidently learnable as follows. On a text for a set with up to two elements, the learner converges to an index for this set using $\left\{W_{0}, W_{1}, W_{2}, \ldots\right\}$ as hypotheses space. The learner does not revise its hypothesis after seeing three elements in the input, in order to obtain confidence.

Note that $\left\{D:|D|=2 \vee\left(|D|=1 \wedge D \subseteq K^{\prime}\right)\right\}$ is an r.e. class. To see this, note that there is a two-place recursive function $g$ with $x \in K^{\prime}$ iff $g(x, y)=1$ for almost all $y$ and $x \notin K^{\prime}$ iff $g(x, y)=0$ for infinitely many $y$. Now let

$$
L_{2\langle x, y\rangle}=\{x, x+y+1\} \text { and }
$$




$$
L_{2\langle x, y\rangle+1}= \begin{cases}\{x, x+z+1\} & \text { if } z \text { is the least number with } \\ \{x\} & z>y \text { and } g(x, z) \neq 1 \\ \text { if } g(x, z)=1 \text { for all } z>y\end{cases}
$$

It is easy to verify that $\left\{L_{0}, L_{1}, \ldots\right\}=\left\{D:|D|=2 \vee\left(|D|=1 \wedge D \subseteq K^{\prime}\right)\right\}$. It is easy to see that $\left\{L_{0}, L_{1}, L_{2}, \ldots\right\}$ is even an indexed family for the given class. Now assume that some confident learner $M$ for $\left\{L_{0}, L_{1}, L_{2}, \ldots\right\}$ uses some hypotheses space $\left\{H_{0}, H_{1}, H_{2}, \ldots\right\}$ with $\left\{H_{0}\right.$, $\left.H_{1}, H_{2}, \ldots\right\}=\left\{L_{0}, L_{1}, L_{2}, \ldots\right\}$. Then one can define the $K$-recursive function $f$ with $f(x)$ being the hypothesis to which $M$ converges on the text $x^{\infty}$. If $x \in K^{\prime}$ then $H_{f(x)}=\{x\}$ as $M$ learns this set. If $x \notin K^{\prime}$ then $H_{f(x)} \neq\{x\}$ as no member of $\left\{H_{0}, H_{1}, H_{2}, \ldots\right\}$ equals $\{x\}$. The test whether $H_{f(x)}=\{x\}$ is also $K$-recursive. This would give a contradiction to $K^{\prime} \mathbb{Z}_{T} K$. Thus there is no class-preserving confident learner for $\left\{L_{0}, L_{1}, L_{2}, \ldots\right\}$.

For conservative learning, a full hierarchy can be established. Note that the following example can be transferred to many related notions like monotonic [22] and non U-shaped learning [2] without giving more insight. Therefore, these learning criteria are not considered in the present work.

Example 11. (a) The class $\{D:|D| \leq 1\}$ is prescribed conservatively but not uniformly conservatively learnable.

(b) The class $\{D:|D|<\infty\}$ is class-preservingly conservatively but not prescribed conservatively learnable.

(c) The class $\left\{D:|D|=2 \vee\left(|D|=1 \wedge D \subseteq K^{\prime}\right)\right\}$ is class-comprisingly conservatively but not class-preservingly conservatively learnable.

Proof. (a) The prescribed learner knows the index $a$ of $\emptyset$ in the given numbering $\left\{H_{0}, H_{1}\right.$, $\left.H_{2}, \ldots\right\}$. So it conjectures $H_{a}$ until a number $x$ occurs in the input and an index $b$ is found with $x \in H_{b}$. Then the learner makes one mind change to $b$ and keeps this index forever. This learner is conservative and correct as $\{x\}$ is the only set in $\left\{H_{0}, H_{1}, H_{2}, \ldots\right\}$ containing $x$. For the second part, let $S$ be a simple set [20], $S^{e}=S \cup\{0,1, \ldots, e\}$, and let $S_{y}^{e}$ denote $S^{e}$ enumerated within $y$ steps, in some effective enumeration (in $e$ ) of $S^{e}$. Define class-preserving hypotheses spaces $\mathcal{H}^{0}, \mathcal{H}^{1}, \mathcal{H}^{2}, \ldots$, where $\mathcal{H}^{e}=\left\{H_{0}^{e}, H_{1}^{e}, H_{2}^{e}, \ldots\right\}$ with $H_{x}^{e}(y)=1$ if $x \in S_{y}^{e}-S_{y-1}^{e}$ and $H_{x}^{e}(y)=0$ if $x \notin S_{y}^{e}-S_{y-1}^{e}$.

If $\{D:|D| \leq 1\}$ is uniformly conservatively learnable, then there exists a recursive family of learners $N_{0}, N_{1}, N_{2}, \ldots$ such that for all $e \in \mathbb{N}, N_{e}$ conservatively learns the class $\{D:|D| \leq 1\}$ with respect to $\mathcal{H}^{e}$. The r.e. set $A=\left\{x\right.$ : for some $e, N_{e}$ outputs $x$ on $\left.\#^{\infty}\right\}$ is infinite (as for all $e, N_{e}$ outputs an index larger than $e$ ) and disjoint to $S$. This contradicts the fact that $S$ is simple.

Note that the hypotheses spaces $\mathcal{H}^{e}$ constructed above are uniformly recursive. Thus, $\{D$ : $|D| \leq 1\}$ is not even uniformly class-preservingly conservatively learnable when the hypotheses spaces must be uniformly recursive. Similar observation holds for part (b), too.

(b) The class of all finite sets is clearly conservatively learnable in the canonical numbering of the finite sets. Now let $I_{0}, I_{1}, I_{2}, \ldots$ be a recursive partition of the natural numbers into intervals 
such that there is a simple set $A$ with $I_{n} \nsubseteq A$ for all $n$. Let $\left\{L_{0}, L_{1}, L_{2}, \ldots\right\}$ be the canonical numbering of the finite sets and let $H_{m}=L_{n}$ for $m \in I_{n}-A$ and $H_{m}=L_{n} \cup\{m+n+t, m+n+t+1\}$ for $m \in I_{n} \cap A$, with $m \in A_{t}-A_{t-1}$. It is easy to see that $\left\{H_{0}, H_{1}, H_{2}, \ldots\right\}$ is also a numbering of all finite sets. Assume now that $M$ is a learner using the hypotheses space $\left\{H_{0}, H_{1}, H_{2}, \ldots\right\}$. Then one defines a recursive function $f$ as follows: $f(x)=b$ for the first $b$ found such that $x \in H_{b}$ and $M\left(x^{k}\right)=b$ for some $k$. As all $H_{b}$ are finite, the set $\{f(0), f(1), f(2), \ldots\}$ contains infinitely many indices and is recursively enumerable. Hence there is an $x$ with $f(x) \in A$. It follows that $\{x\} \subset H_{f(x)}$ as $H_{f(x)}$ contains at least two elements. So the learner $M$ overgeneralizes on $x^{k}$ and is not conservative.

(c) In Theorem 10, it has been shown that the class $\left\{D:|D|=2 \vee\left(|D|=1 \wedge D \subseteq K^{\prime}\right)\right\}$ is an r.e. class. The class-comprising confident learner given there is also conservative. Now assume that some conservative learner $M$ for this class uses some class-preserving hypotheses space $\left\{H_{0}\right.$, $\left.H_{1}, H_{2}, \ldots\right\}$. Then one can again define $f(x)$, this time only partial-recursive, to be the $b$ found such that $M$ outputs $b$ on the text $x^{\infty}$ and $x \in H_{b}$. Now $x \in K^{\prime}$ iff $f(x)$ is defined and $H_{f(x)}=\{x\}$. This condition can be checked with oracle $K$ although $K^{\prime} \mathbb{Z}_{T} K$. From this contradiction follows that there is no class-preserving conservative learner for $\left\{D:|D|=2 \vee\left(|D|=1 \wedge D \subseteq K^{\prime}\right)\right\}$.

\section{$3 \quad$ Vacillatory and Behaviourally Correct Learning}

For vacillatory and behaviourally correct learning, a strict hierarchy from prescribed to classpreserving to class-comprising learning can be established. It remains open whether uniform learning is more restrictive than prescribed learning.

Theorem 12. Let $L_{2 a}=\{\langle a, b\rangle: b \in \mathbb{N}\}$ and $L_{2 a+1}=\left\{\langle a, b\rangle: b \leq\left|W_{a}\right|\right\}$. Then $\left\{L_{0}, L_{1}\right.$, $\left.L_{2}, \ldots\right\}$ is uniformly behaviourally correct learnable and class-preservingly vacillatorily learnable but neither prescribed vacillatorily learnable nor class-comprisingly explanatorily learnable.

Proof. Assume that $\left\{H_{0}, H_{1}, H_{2}, \ldots\right\}=\left\{L_{0}, L_{1}, L_{2}, \ldots\right\}$ and $W_{e}=\left\{\langle b, x\rangle: x \in H_{b}\right\}$. Let $s$ be the length and $D$ be the content of the input. Now a learner $M_{e}$ is constructed. $M_{e}$ first computes the sets

$-A=\left\{c \leq s: D=H_{c, s}\right\}$ and

$-B=\left\{c \leq s: D \cap H_{c, s} \neq \emptyset\right\}$

then $M_{e}$ follows the first of the following cases which applies:

- If $D=\emptyset$ then $M_{e}$ outputs?;

- If $A \neq \emptyset$ then $M_{e}$ outputs $\min (A)$;

- If $B \neq \emptyset$ then $M_{e}$ outputs some $c \in B$ for which $H_{c, s}$ has largest number of elements;

- Otherwise $M_{e}$ repeats the previous conjecture.

The first case, together with the last, make sure that $M_{e}$ is total, starts with ? and never returns to ? once it has taken another hypothesis. Assume now that $M_{e}$ sees a text for a language $H_{b} \in\left\{L_{2 a}, L_{2 a+1}\right\}$ and that $b$ is the least index of $H_{b}$ in $\left\{H_{0}, H_{1}, H_{2}, \ldots\right\}$. Furthermore, assume that so much data has been observed such that the following four conditions hold: 
$-s \geq b$

- The datum $\langle a, 0\rangle$ is in both, $D$ and $H_{b, s}$;

- If $H_{b} \neq L_{2 a+1}$ then $\left|H_{b, s}\right|>\left|L_{2 a+1}\right|$ and $|D|>\left|L_{2 a+1}\right|$;

- If $H_{b}$ is finite then $H_{b}=H_{b, s}=D$ and, for all $d<b$ and $t \geq s, H_{d, t} \neq D$.

Note that $D \neq \emptyset$ and $B \neq \emptyset$ and therefore $M_{e}$ outputs a hypothesis $c$ different from ?. Now it is shown that $H_{c}=H_{b}$ : First note that $\langle a, 0\rangle \in D$ and $b \in B$, hence the algorithm chooses $c$ either by the second or the third condition in the algorithm. It follows that $H_{c}=L_{2 a}$ or $H_{c}=L_{2 a+1}$. If $H_{b}$ is finite, it follows directly from the learning algorithm that $b=\min (A)$ for the set $A$ considered there and hence $c=b$. If $H_{b}$ is infinite and $L_{2 a+1}$ is finite, then $\left|H_{c}\right| \geq\left|H_{b, s}\right|>\left|L_{2 a+1}\right|$ and $H_{c}=L_{2 a}=H_{b}$. If $H_{b}$ and $L_{2 a+1}$ are both infinite then $H_{b}=L_{2 a}=L_{2 a+1}$ and $H_{c}=H_{b}$. So $M_{e}$ is a behaviourally correct learner for $\left\{L_{0}, L_{1}, L_{2}, \ldots\right\}$ using the hypotheses space $\left\{H_{0}, H_{1}\right.$, $\left.H_{2}, \ldots\right\}$.

To see that $\left\{L_{0}, L_{1}, L_{2}, \ldots\right\}$ is class-preservingly vacillatorily learnable, take $H_{b}=L_{b}$ for all $b$. For each language there are at most 2 indices in $\left\{H_{0}, H_{1}, H_{2}, \ldots\right\}$ and therefore the above described behaviorally correct learner is also a vacillatory one.

To see that $\left\{L_{0}, L_{1}, L_{2}, \ldots\right\}$ is not prescribed vacillatory learnable, one constructs a suitable hypotheses space as follows:

$$
H_{\langle a, b\rangle}= \begin{cases}L_{2 a+1} & \text { if } b=\min \left(\left\{s:\left|W_{a, s}\right|=\left|W_{a}\right|\right\}\right) \\ L_{2 a} & \text { otherwise. }\end{cases}
$$

For each $a$ there is a $b$ with $H_{\langle a, b\rangle}=L_{2 a+1}$; if $W_{a}$ is finite then one can take $b$ as the minimum of the nonempty set $\left\{s:\left|W_{a, s}\right|=\left|W_{a}\right|\right\}$; if $W_{a}$ is infinite then one can take $b=0$. The reason for the latter case is that then $L_{2 a}=L_{2 a+1}$. Furthermore, all but at most one of the $b$ satisfy $L_{2 a}=H_{\langle a, b\rangle}$. Hence $\left\{H_{0}, H_{1}, H_{2}, \ldots\right\}$ is a hypotheses space for $\left\{L_{0}, L_{1}, L_{2}, \ldots\right\}$. If there were a prescribed vacillatory learner using $\left\{H_{0}, H_{1}, H_{2}, \ldots\right\}$ as the hypothesis space then there would also be a $K$-recursive function $f$ such that $f(a)$ is the maximal element output by this learner on the canonical text for $L_{2 a+1}$. It would follow that $W_{a}$ is finite iff $W_{a, f(a)}=W_{a}$; note that $f(a) \geq\langle a, b\rangle \geq b$ for the least $b$ such that $L_{2 a+1}=H_{\langle a, b\rangle}$. But then a $K$-recursive procedure could check, given $a$, whether $W_{a}$ is finite. As such a procedure does not exist $[21],\left\{L_{0}, L_{1}\right.$, $\left.L_{2}, \ldots\right\}$ is not vacillatorily learnable with respect to the hypotheses space $\left\{H_{0}, H_{1}, H_{2}, \ldots\right\}$.

As just seen, $\left\{L_{0}, L_{1}, L_{2}, \ldots\right\}$ is not prescribed vacillatorily learnable and hence also not prescribed explanatorily learnable. It follows using Theorem 9 that $\left\{L_{0}, L_{1}, L_{2}, \ldots\right\}$ is also not class-comprisingly explanatorily learnable.

Theorem 13. For all $a, b$ let

$$
L_{\langle a, b\rangle}= \begin{cases}\{\langle a, c\rangle: c \in \mathbb{N}\} & \text { if } b=0 ; \\ \left\{\langle a, c\rangle: c \leq\left|W_{a}\right|\right\} & \text { if } b=1 ; \\ \left\{\langle a, c\rangle: c \leq\left|W_{a, d}\right|\right\} \cup\left\{\left\langle a+1,\left|W_{a, d}\right|+e+1\right\rangle\right\} & \text { if } b=2+\langle d, e\rangle .\end{cases}
$$

The class $\left\{L_{0}, L_{1}, L_{2}, \ldots\right\}$ is class-preservingly behaviourally correct learnable but not prescribed behaviourally correct learnable. 
Proof. Recall that $\left|W_{a, d}\right| \leq d+1$ for all $d$. It is easy to see that $\left\{L_{0}, L_{1}, L_{2}, \ldots\right\}$ is a uniformly r.e. class. Assume that an input of length $s$ and content $D$ is given. A behaviourally correct learner takes now the first case which applies.

- If there is a pair $\langle a, b\rangle$ such that $\langle a+1, a+b+2\rangle<s$ and $L_{\langle a, b\rangle, s}=D$ then output $\langle a, b\rangle$ for the least pair where these conditions are true.

- If there is an $a$ such that $\{\langle a, 0\rangle\} \subseteq D \subseteq L_{\langle a, 0\rangle}$ then output $\langle a, 0\rangle$.

- Otherwise output?.

In this context it is assumed that for $b>1$ and $s>\langle a+1, a+b+2\rangle, L_{\langle a, b\rangle, s}=L_{\langle a, b\rangle}$ as one can compute all members directly from the parameters $a, b$. It is easy to see that this learner succeeds on all finite sets from $\left\{L_{0}, L_{1}, L_{2}, \ldots\right\}$. So assume that an infinite set $L_{\langle a, 0\rangle}$ is given. If $L_{\langle a, 1\rangle}=L_{\langle a, 0\rangle}$ then the learner will eventually vacillate between these two indices. If $L_{\langle a, 1\rangle} \subset L_{\langle a, 0\rangle}$ then $L_{\langle a, 1\rangle}$ is finite and as the learner eventually sees an element of $L_{\langle a, 0\rangle}-L_{\langle a, 1\rangle}$, it will converge to $\langle a, 0\rangle$. So $\left\{L_{0}, L_{1}, L_{2}, \ldots\right\}$ is class-preservingly behaviourally correct learnable.

Now a hypotheses space is constructed using which $\left\{L_{0}, L_{1}, L_{2}, \ldots\right\}$ cannot be behaviourally correct learned. For all $a, b$ let

$$
\begin{aligned}
H_{\langle a, 0\rangle} & =L_{\langle a, 0\rangle} ; \\
H_{\langle a, 2 b+1\rangle} & =L_{\langle a, b+2\rangle} ; \\
H_{\langle a, 2 b+2\rangle} & = \begin{cases}\left\{\langle a, c\rangle: c \leq\left|W_{a, b}\right|\right\} & \text { if } W_{a, b}=W_{a} ; \\
\left\{\langle a, c\rangle: c \leq\left|W_{a, b}\right|\right\} \cup\left\{\left\langle a+1,\left|W_{a, b}\right|+s+1\right\rangle\right\} & \text { if } s \text { is the least number }\end{cases}
\end{aligned}
$$

It is easy to check that this class is an indexed family, that is, $\left\{H_{0}, H_{1}, H_{2}, \ldots\right\}$ is uniformly recursive. Thus, if one could behaviourally correct learn $\left\{L_{0}, L_{1}, L_{2}, \ldots\right\}$ using $\left\{H_{0}, H_{1}, H_{2}, \ldots\right\}$ as the hypotheses space, one could also explanatorily learn $\left\{L_{0}, L_{1}, L_{2}, \ldots\right\}$ using $\left\{H_{0}, H_{1}\right.$, $\left.H_{2}, \ldots\right\}$ (this folklore result is based on the observation that, for hypotheses space being an indexed family, the mind changes can be delayed until it can be verified that the later hypothesis differs from the earlier one). Using Theorem 9, this would imply that the class from Theorem 12 (which is contained in $\left\{L_{0}, L_{1}, L_{2}, \ldots\right\}$ ) is prescribed explanatorily learnable and hence prescribed vacillatory learnable. This contradicts Theorem 12 . So $\left\{L_{0}, L_{1}, L_{2}, \ldots\right\}$ is not prescribed behaviourally correct learnable.

Corollary 14. Let $\left\{L_{0}, L_{1}, L_{2}, \ldots\right\}$ be as in Theorem 13. Then $\left\{L_{0}, L_{1}, L_{2}, \ldots\right\} \cup\{\mathbb{N}\}$ is classpreserving behaviourally correct learnable. Furthermore, no $\left\{F_{0}, F_{1}, F_{2}, \ldots\right\} \supseteq\left\{L_{0}, L_{1}, L_{2}, \ldots\right\} \cup$ $\{\mathbb{N}\}$ is prescribed behaviourally correct learnable.

Proof. The class-preserving behaviourally correct learner for $\left\{L_{0}, L_{1}, L_{2}, \ldots\right\}$ from Theorem 13 can easily be extended to one for $\left\{L_{0}, L_{1}, L_{2}, \ldots\right\} \cup\{\mathbb{N}\}$. Let $\left\{H_{0}, H_{1}, H_{2}, \ldots\right\}$ be the uniformly recursive hypotheses space for $\left\{L_{0}, L_{1}, L_{2}, \ldots\right\}$ from Theorem 13 . Now define

$$
G_{0}=\mathbb{N}
$$




$$
\begin{aligned}
G_{2 a+1} & =H_{a} ; \\
G_{2\langle a, b\rangle+2} & = \begin{cases}\mathbb{N} & \text { if there are } c, e, t \text { with } t>b+c+(e+7)^{2} \text { and } F_{a, t} \neq \emptyset \text { and } \\
& \text { either } F_{a, t}=H_{e} \cap\{0,1, \ldots, t\} \\
& \text { or }\{\langle c, 0\rangle,\langle c, 1\rangle, \ldots,\langle c, b\rangle\} \subseteq F_{a, t} \subseteq\{\langle c, 0\rangle,\langle c, 1\rangle, \ldots\} ; \\
F_{a} & \text { otherwise. }\end{cases}
\end{aligned}
$$

Note that the bound $(e+7)^{2}$ is used in the formula above to ensure the condition $\max \left(H_{\langle a, 2 b+1\rangle}\right) \leq$ $(\langle a, 2 b+1\rangle+7)^{2}$ for all $a, b$ which is used implicitly in Case (e) below.

Clearly $\left\{L_{0}, L_{1}, L_{2}, \ldots\right\} \cup\{\mathbb{N}\} \subseteq\left\{G_{0}, G_{1}, G_{2}, \ldots\right\}$. Furthermore, if $\emptyset \in\left\{F_{0}, F_{1}, F_{2}, \ldots\right\}$ then $\emptyset \in\left\{G_{0}, G_{1}, G_{2}, \ldots\right\}$. Assume now that $F_{a}$ is not in $\left\{L_{0}, L_{1}, L_{2}, \ldots\right\} \cup\{\emptyset, \mathbb{N}\}$. Let $c$ be the least number such that there is some $d$ with $\langle c, d\rangle \in F_{a}$; fix this $d$ as well. There are five cases.

Case (a): $F_{a}$ contains two elements $\left\langle c^{\prime}, d^{\prime}\right\rangle,\left\langle c^{\prime \prime}, d^{\prime \prime}\right\rangle$ with $\left.c^{\prime}\right\rangle c, d^{\prime \prime} \geq d^{\prime}$ and $\left\langle c^{\prime}, d^{\prime}\right\rangle \neq\left\langle c^{\prime \prime}, d^{\prime \prime}\right\rangle$. Then let $b$ be so large that $\langle c, d\rangle,\left\langle c^{\prime}, d^{\prime}\right\rangle \in F_{a, b}$. Now it follows that $F_{a, b} \nsubseteq H_{e}$ for all $e$ and $G_{2\langle a, b\rangle+2}=F_{a}$.

Case (b): $F_{a}$ is the union of $\left\{\left\langle c^{\prime}, d^{\prime}+1\right\rangle\right\}$ with a subset of $\left\{\langle c, 0\rangle,\langle c, 1\rangle, \ldots,\left\langle c, d^{\prime}\right\rangle\right\}$ for some $c^{\prime}, d^{\prime}$ with $c^{\prime}>c$. It is easy to verify that $F_{a} \neq H_{e} \cap\left\{0,1, \ldots,\left\langle c^{\prime}, d^{\prime}+1\right\rangle\right\}$ for all $e$. Let $b$ be so large that $F_{a, b}=F_{a}$ and $b \geq\left\langle c^{\prime}, d^{\prime}+1\right\rangle$. Then, for all $t>b, F_{a, t} \neq H_{e} \cap\{0,1, \ldots, t\}$. Hence $G_{2\langle a, b\rangle+2}=F_{a}$.

Case (c): $F_{a} \subset\{\langle c, 0\rangle,\langle c, 1\rangle, \ldots\}$ and there are $d^{\prime}, d^{\prime \prime}$ with $d^{\prime}\left\langle d^{\prime \prime},\left\langle c, d^{\prime}\right\rangle \notin F_{a}\right.$ and $\left\langle c, d^{\prime \prime}\right\rangle \in F_{a}$. Let $b$ be so large that $\left\langle c, d^{\prime \prime}\right\rangle \in F_{a, b}$ and $b>\left\langle c, d^{\prime \prime}\right\rangle$. Then, for all $e$ and all $t>b, F_{a, t} \neq$ $H_{e} \cap\{0,1, \ldots, t\}$. Furthermore, the condition $\{\langle c, 0\rangle,\langle c, 1\rangle, \ldots,\langle c, b\rangle\} \subseteq F_{a, t} \subseteq\{\langle c, 0\rangle,\langle c, 1\rangle, \ldots\}$ does not hold. Hence $G_{2\langle a, b\rangle+2}=F_{a}$.

Case $(\mathrm{d}): F_{a}=\left\{\langle c, 0\rangle,\langle c, 1\rangle, \ldots,\left\langle c, d^{\prime}\right\rangle\right\}$ and $d^{\prime}>\left|W_{c}\right|$. Then there is no $H_{e}$ with $F_{a}=$ $H_{e} \cap\left\{0,1, \ldots,\left\langle c, d^{\prime}+1\right\rangle\right\}$. Taking $b$ so large that $F_{a, b}=F_{a}$ and $b \geq\left\langle c, d^{\prime}+1\right\rangle$, the condition $\{\langle c, 0\rangle,\langle c, 1\rangle, \ldots,\langle c, b\rangle\} \subseteq F_{a, t} \subseteq\{\langle c, 0\rangle,\langle c, 1\rangle, \ldots\}$ becomes false and thus $G_{2\langle a, b\rangle+2}=F_{a}$.

Case (e): $F_{a}=\left\{\langle c, 0\rangle,\langle c, 1\rangle, \ldots,\left\langle c, d^{\prime}\right\rangle\right\}$ and $d^{\prime}<\left|W_{c}\right|$. Let $s$ be so large that $d^{\prime}+1=\left|W_{c, s}\right|$ and take $b$ so large that $\left\langle c+1,\left|W_{c, s}\right|+s+1\right\rangle \leq b,\left\langle c, d^{\prime}+1\right\rangle \leq b$ and $F_{a, b}=F_{a}$. Then $F_{a, t} \neq H_{e} \cap$ $\{0,1, \ldots, t\}$ for all $e$ and $t>b+(e+7)^{2}$. Furthermore, the condition $\{\langle c, 0\rangle,\langle c, 1\rangle, \ldots,\langle c, b\rangle\} \subseteq$ $F_{a, t} \subseteq\{\langle c, 0\rangle,\langle c, 1\rangle, \ldots\}$ does not hold. Hence $G_{2\langle a, b\rangle+2}=F_{a}$.

Note that the sets in $\left\{H_{0}, H_{1}, H_{2}, \ldots\right\}$ have only odd indices in the numbering $\left\{G_{0}, G_{1}\right.$, $\left.G_{2}, \ldots\right\}$. Hence, given a behaviourally correct learner $M$ for $\left\{F_{0}, F_{1}, F_{2}, \ldots\right\}$ using the hypotheses space $\left\{G_{0}, G_{1}, G_{2}, \ldots\right\}$, one can build the following new learner $N$ for $\left\{H_{0}, H_{1}, H_{2}, \ldots\right\}$ using the hypotheses space $\left\{H_{0}, H_{1}, H_{2}, \ldots\right\}$ itself:

$$
N(\sigma)= \begin{cases}\frac{1}{2}(M(\sigma)-1) & \text { if } M(\sigma) \text { is odd; } \\ ? & \text { if } M(\sigma) \text { is even or ?. }\end{cases}
$$

This contradicts Theorem 13 which showed that such a learner does not exist.

For the next result, let $I_{n}=\left\{2^{n}-1,2^{n}, 2^{n}+1, \ldots, 2^{n+1}-3,2^{n+1}-2\right\}$ form a partition of the natural numbers into intervals of length $2^{n}$ and let $C$ denote the plain Kolmogorov complexity [17]: $C(x)=\min \left(\left\{n: \exists y \in I_{n}\left[\varphi_{y}(0)=x\right]\right\}\right)$. Furthermore, let

$$
A=\left\{m: \exists n\left[m \in I_{n} \wedge C(m)<0.4 n\right]\right\} \text { and }
$$




$$
B=\left\{m: \exists n\left[m \in I_{n} \wedge C(m)>0.8 n\right]\right\}
$$

be the sets of numbers of small and large Kolmogorov complexity, respectively.

Theorem 15. Let $A$ and $B$ be the sets of numbers of small and large Kolmogorov complexity as above. Then the class consisting of $\mathbb{N}, A$ and all sets $A \cup\{b\}$ with $b \in B$ is uniformly r.e. and is class-comprisingly but not class-preservingly behaviourally correct learnable.

Proof. Note that $A$ is recursively enumerable and $B$ is co-r.e.; an indexing of the class is now given by fixing one index $a \in A$ and then letting $L_{a}=A, L_{b}=A \cup\{b\}$ for all $b \in B$ and $L_{b}=\mathbb{N}$ for all $b \in \mathbb{N}-B-\{a\}$.

Note that $0 \notin A \cup B$. Hence $\mathbb{N}$ is the only member of $\left\{L_{0}, L_{1}, L_{2}, \ldots\right\}$ containing 0 . Furthermore, let $D_{0}, D_{1}, \ldots$ be a canonical enumeration of all finite sets. Now let

$$
H_{b}= \begin{cases}\mathbb{N} & \text { if } 0 \in D_{b} \\ D_{b} \cup A & \text { if } 0 \notin D_{b}\end{cases}
$$

Furthermore, one can build a behaviourally correct learner using the hypotheses space $\left\{H_{0}\right.$, $\left.H_{1}, H_{2}, \ldots\right\}$ by conjecturing $H_{b}$ for the unique $b$ with $D_{b}=\operatorname{content}(\sigma)$ on input $\sigma$. It is easy to verify that this learner succeeds on all languages in $\left\{H_{0}, H_{1}, H_{2}, \ldots\right\}$. Therefore $\left\{L_{0}, L_{1}\right.$, $\left.L_{2}, \ldots\right\}$ is class-comprisingly behaviourally correct learnable.

Now assume that $M$ is a class-preserving behaviourally correct learner for $\left\{L_{0}, L_{1}, L_{2}, \ldots\right\}$. There is a family $T_{0}, T_{1}, \ldots$ of texts and an $n$ such that

- $T_{x}[n]$ is a fixed semantic locking sequence for $M$ on $A$;

$-T_{x}(n)=x$

- for all $x$, the subsequence $T_{x}(n+1), T_{x}(n+2), T_{x}(n+3), \ldots$ of $T_{x}$ is the same recursive enumeration of $A$.

Now one defines two sets $X$ and $Y$ according to the behaviour of $M$ on $T_{x}$.

- $X$ is the set of all $x$ such that, for some $m>n, M\left(T_{x}[m]\right)$ conjectures a set containing $x$;

- $Y$ is the set of all $x$ such that, for some $m>n, M\left(T_{x}[m]\right)$ conjectures a set containing 0 .

Both sets are recursively enumerable. The set $Y$ is disjoint to $A$ as, for all $x \in A$ and all $m>n$, $M\left(T_{x}[m]\right)$ is an index of $A$. As $A$ is a simple set [17], $Y$ is finite. As $A \cup B \subseteq X \subseteq A \cup B \cup Y$, the set $A \cup B$ is recursively enumerable. For each sufficiently large $n$, at least half of elements of $I_{n}$ are in $A \cup B$. Now let $J_{n}$ be the first $2^{0.6 n}$ elements of $I_{n}$ to be enumerated into $A \cup B$. The $J_{n}$ are uniformly r.e. and due to Kolmogorov-complexity considerations, for all sufficiently large $n$, $J_{n} \cap B=\emptyset$. Hence $J_{n} \subseteq A \cap I_{n}$ in contradiction to the fact that $\left|A \cap I_{n}\right| \leq 2^{0.4 n}$. This shows that the learner $M$ cannot exist and $\left\{L_{0}, L_{1}, L_{2}, \ldots\right\}$ is not class-preservingly behaviourally correct learnable.

Theorem 16. There exists an r.e. class $\mathcal{L}$ which is class-comprisingly but not class-preservingly vacillatorily learnable. 
Proof. In the following, let $\langle x, y, z\rangle$ denote $\langle x,\langle y, z\rangle\rangle$. The class $\mathcal{L}$ will be a suitable subclass of the following:

$$
\begin{aligned}
L_{\langle e, 2 a\rangle} & =\{\langle e, a, b\rangle: b \in \mathbb{N}\} \\
L_{\langle e, 2 a+1\rangle} & =\left\{\langle e, a, b\rangle: b \leq\left|W_{a}\right|\right\}
\end{aligned}
$$

The proof of Theorem 12 can be adapted to show that $\mathcal{L}$ is class-comprisingly vacillatorily learnable. Let

$$
G_{a}^{e}=\left\{x:\langle a, x\rangle \in W_{e}\right\}
$$

be the $a$-th set in the $e$-th recursively enumerable hypotheses space. Now define a limit-recursive predicate $P$ as follows:

$$
P(e, a, b)= \begin{cases}1 & \text { if } \forall c<b\left[G_{a}^{e} \neq\{\langle e, a, d\rangle: d \leq b\}\right] \\ 0 & \text { if } \exists c<b\left[G_{a}^{e}=\{\langle e, a, d\rangle: d \leq b\}\right]\end{cases}
$$

and let

$$
\mathcal{L}=\left\{L_{\langle e, 2 a\rangle}: e, a \in \mathbb{N}\right\} \cup\left\{L_{\langle e, 2 a+1\rangle}: e, a \in \mathbb{N} \wedge\left|W_{a}\right|<\infty \wedge P\left(e, a,\left|W_{a}\right|\right)\right\}
$$

It is easy to verify that $\mathcal{L}$ is an r.e. class. Suppose by way of contradiction that $\left\{H_{0}, H_{1}, H_{2}, \ldots\right\}=$ $\mathcal{L}$ and a learner $M$ vacillatorily learns $\mathcal{L}$ using $\left\{H_{0}, H_{1}, H_{2}, \ldots\right\}$ as hypotheses space. Let $e$ be such that $W_{e}=\left\{\langle c, x\rangle: x \in H_{c}\right\}$. So $G_{c}^{e}=H_{c}$ for all $c$. Note that $P\left(e, a,\left|W_{a}\right|\right)=1$ for all $a$ where $W_{a}$ is finite; the reason is the following chain of implications: $P\left(e, a,\left|W_{a}\right|\right)=0 \Rightarrow \exists c<\left|W_{a}\right|$ $\left[G_{c}^{e}=L_{\langle e, 2 a+1\rangle}\right] \Rightarrow L_{\langle e, 2 a+1\rangle} \in \mathcal{L} \Rightarrow P\left(e, a,\left|W_{a}\right|\right)=1$. Thus $L_{\langle e, 2 a+1\rangle} \in \mathcal{L}$ whenever $W_{a}$ is finite. Furthermore, $L_{\langle e, 2 a+1\rangle} \in \mathcal{L}$ whenever $W_{a}$ is infinite, as then $L_{\langle e, 2 a+1\rangle}=L_{\langle e, 2 a\rangle}$. Thus $M$ learns $L_{\langle e, 2 a+1\rangle}$ for all $a$.

Let $T_{a}$ be a text for $L_{\langle e, 2 a+1\rangle}$ uniformly recursive in the parameter $a$. Then $M$ on $T_{a}$ outputs only finitely many indices; let $g(a)$ be the greatest among these indices. It follows that $g(a) \geq\left|W_{a}\right|$ whenever $W_{a}$ is finite; the reason is that $P\left(e, a,\left|W_{a}\right|\right)=1$, thus no $H_{c}$ with $c<\left|W_{a}\right|$ equals $L_{\langle e, 2 a+1\rangle}$. This gives that $W_{a}$ is finite iff $\left|W_{a}\right| \leq g(a)$. As $g \leq_{T} K,\left\{a:\left|W_{a}\right|<\infty\right\} \leq_{T} K$, a contradiction.

\section{Prudence for Behaviourally Correct Learning}

Osherson, Stob and Weinstein [19] were interested in the question whether every learnable class is prudently learnable. Fulk [9] showed that every explanatory learnable class is prudently explanatory learnable. Jain and Sharma [12] showed the corresponding result for vacillatory learning. The next theorem shows this result for behaviourally correct learning. In 1988, Kurtz and Royer [14] had claimed to have this result, but their proof had a bug and the problem had remained open since then. Furthermore, the construction of the prudent learner in the next theorem is effective in the original learner. It is still open whether prudence for explanatory and vacillatory learning can be effectivized. 
Theorem 17. If $\mathcal{L}$ is a (not necessarily uniformly r.e.) behaviourally correct learnable class then $\mathcal{L}$ is a subclass of an r.e. class which is class-preservingly behaviourally correct learnable.

Proof. For any set $A$, let $T_{A}$ be the ascending text which is given by $T_{A}(x)=x$ for all $x \in A$ and $T_{A}(x)=\#$ for all $x \notin A$. Furthermore, let $\delta_{\emptyset}$ be the empty string and $\delta_{A}=T_{A}[\max (A)+1]$ for all finite non-empty sets $A$. For example, $\delta_{\{0,2,3\}}=0 \# 23$.

There is a behaviourally correct learner for the class $\mathcal{L}$ using the acceptable numbering $\left\{W_{0}, W_{1}, W_{2}, \ldots\right\}$ as hypotheses space and satisfying the following constraints:

- $M$ is consistent, that is, $\operatorname{content}(\sigma) \subseteq W_{M(\sigma)}$ for all $\sigma$;

- $M$ is rearrangement-independent, that is, $W_{M(\sigma)}=W_{M(\tau)}$ whenever $\sigma, \tau$ have the same content and length;

- $W_{M(\sigma)}$ is finite whenever $\sigma$ is not a semantical locking sequence for $M$ on $W_{M(\sigma)}$.

Kurtz and Royer [14] showed that the first two conditions can be satisfied and such a learner can be found effectively from any given learner. The third condition can also be effectively added since the complement of the set of semantical locking sequences is $K$-r.e.; that is, $\sigma$ is not a semantical locking sequence iff there is a $\tau$ in $\left(W_{M(\sigma)} \cup\{\#\}\right)^{*}$ and an $x \in \mathbb{N}$ with $x \in W_{M(\sigma \tau)} \Leftrightarrow x \notin W_{M(\sigma)}$. For that reason, $M$ is a behaviourally correct learner for all infinite sets for which some index is output by $M$. So, to prove the theorem, one has mainly to take care of finite sets.

Now the following new learner $N$ is constructed. $N$ is defined by mapping $\sigma$ to a hypothesis $H_{\sigma}$; thus the hypotheses space is given directly instead of $N . H_{\sigma}$ takes the first case which applies.

Intuitively, Case (2) below handles learnability of all infinite sets behaviourally learnt by $M$, besides ensuring some nice properties of $H_{\delta_{D}}$ (main one being that $H_{\delta_{D}}$ does not contain any element in $\{0,1, \ldots, \max D\}-D$ or it follows $H_{\delta_{F}}$ for some appropriate proper subset $F$ of $D$ ). During this process, Case (2) might introduce some finite sets into the hypotheses space. Case (3) ensures learnability of all the finite sets learnt by $M$ as well as those introduced by Case (2) in the hypotheses space - for all other finite sets $D$, Case (3) would mimic Case (2). Case (4) is just to map the remaining sequences to one of Case (2) or (3).

We now formally define $H_{\sigma}$.

Case (1): $H_{\# s}=\emptyset$ for all $s$.

Case (2): $H_{\delta_{D}}$ first enumerates all elements of $D$.

Let $D^{\prime}=\{0,1, \ldots, \max (D)\}-D$. Let $S=\left\{s: W_{M\left(\delta_{D} \#^{\max (D)}\right), s} \cap D^{\prime}=\emptyset\right\}$.

For all $s \in S$, enumerate all elements of $W_{M\left(\delta_{D} \# \max (D)\right), s}$ into $H_{\delta_{D}}$.

If $W_{M\left(\delta_{D} \# \max (D)\right)} \cap D^{\prime}$ is not empty, let $s=\max (S)$, let $E=D \cup W_{M\left(\delta_{D} \#^{\max (D)}\right), s}$, let $x=$ $\min \left(W_{M\left(\delta_{D} \#^{\max (D)}\right), s+1} \cap D^{\prime}\right)$ and let $F=D \cap\{0,1, \ldots, x\}$.

Now, if $H_{\delta_{F}} \supseteq E$ then $H_{\delta_{D}}=H_{\delta_{F}}$ else $H_{\delta_{D}}=E$.

Case (3): $H_{\delta_{D} \#^{s}}$ with $s>0$ is defined as follows. If there is an $x$ such that $H_{\delta_{E_{x}}, s}=H_{\delta_{E_{x}}}=D$ for the set $E_{x}=D \cap\{0,1, \ldots, x\}$ or if $W_{M\left(\delta_{D} \#^{t}\right)}=D$ for all $t \geq s$ then $H_{\delta_{D} \#^{s}}=D$ else $H_{\delta_{D} \#^{s}}=H_{\delta_{D}}$.

Case (4): $H_{\sigma}=H_{\delta_{D} \#^{s}}$ if $H_{\sigma}$ is not defined by Cases (1), (2), (3), s= $\max (\{|\sigma|-\max (D)-1,0\})$ and $D=\operatorname{content}(\sigma)$. 
Note that the only infinite sets in the hypotheses space are the ones which are conjectured by $M$. So $M$ learns all the infinite sets in the hypotheses space. Furthermore, for any $A$ in the hypotheses space, if $E_{x}=\{0,1, \ldots, x\} \cap A$ and $\delta_{E_{x}} \#^{\max \left(E_{x}\right)}$ is a semantic locking sequence for $M$ on $A$, then for all finite $D$ such that $E_{x} \subseteq D \subseteq A, H_{\delta_{D}}=A$. This can be easily seen by induction on cardinality of $D-E_{x}$, as in Case (2), either $H_{\delta_{D}}$ is made equal to $A$ or $H_{\delta_{D}}$ would simulate $H_{\delta_{F}}$ for some $F$ such that $E_{x} \subseteq F \subset D$.

It will be shown first that the hypotheses space covers all sets learned by $M$ and then it will be shown that all sets in the hypotheses space are learned by $N$.

Clearly if $M$ learns a finite set $D$ then $H_{\delta_{D} \#^{s}}=D$ for almost all $s$. Now consider an infinite set $A$ learned by $M$. Let $E_{x}=A \cap\{0,1,2, \ldots, x\}$ for all $x$. As $M$ learns $A$ there is a semantic locking sequence $\tau$ for $M$ on $A$. Now let $x \in A$ be such that $x>|\tau|+\max (\operatorname{content}(\tau))$. Then, for the sequence $\delta_{E_{x}} \#^{\max \left(E_{x}\right)}$, there is an $\eta \in\left(E_{x} \cup\{\#\}\right)^{*}$ such that $|\tau \eta|=\left|\delta_{E_{x}} \#^{\max \left(E_{x}\right)}\right|$ and $\operatorname{content}(\tau \eta)=\operatorname{content}\left(\delta_{E_{x}} \#^{\max \left(E_{x}\right)}\right)=E_{x}$. As $M$ is rearrangement-independent, one has that $W_{M\left(\delta_{E_{x}} \# \max \left(E_{x}\right)\right)}=A$. Hence $H_{\delta_{E_{x}}}=A$ as well. This completes the first part of the verification.

For the second part of the verification consider any set $A$ occurring in the hypotheses space of $N$. There are three cases, those where $A$ is empty, where $A$ is finite but not empty and where $A$ is infinite.

Case (a): $A=\emptyset . N$ learns $A$ as $H_{\#^{s}}=\emptyset$ for all $s$ by Case (1) in the algorithm to enumerate the hypotheses space.

Case (b): $A$ is finite but not empty. Let $D$ be smallest set such that $H_{\delta_{D} \#^{s}}=A$ for some $s$. By Case (1) in the algorithm for $H_{\sigma}, D$ is not empty.

Assume the subcase $A=H_{\delta_{D} \#^{s}} \subset H_{\delta_{D}}$. By Case (3) and $D$ being smallest set such that $H_{\delta_{D} \#^{t}}=A$ for some $t$, this can happen only if $A=D$ and $W_{M\left(\delta_{D} \#^{t}\right)}=D$ for all $t \geq s$. So $H_{\delta_{D} \#^{t}}=D$ for all $t \geq s$ and hence $N$ learns $A$ in this subcase as well.

Assume the subcase $A=H_{\delta_{D} \#^{s}}=H_{\delta_{D}}$. Hence, by Case (2) it follows that there is no element in $A-D$ below $\max (D)$ since otherwise $H_{\delta_{F}}=A$ for some $F \subset D$. Thus, $D=$ $A \cap\{0,1, \ldots, \max (D)\}$. Therefore, $H_{\delta_{A} \#^{t}}=A$ for almost all $t$ and $N$ learns $A$.

Case (c): $A$ is infinite. Again, let $E_{z}=A \cap\{0,1, \ldots, z\}$ for all $z$. As $M$ is rearrangementindependent, there is a semantic locking sequence for $M$ on $A$ of the form $\delta_{E_{x}} \#{ }^{\max \left(E_{x}\right)}$. Hence only finitely many sets $H_{\delta_{E_{z}}}$ are finite. So there is an $y \in A$ such that $y>x$ and $y$ is greater than all elements of these finite sets $H_{\delta_{E_{z}}}$. Let $F$ be any finite set with $E_{y} \subseteq F \subseteq A$. Let $G_{z}=F \cap\{0,1, \ldots, z\}$. If $z \geq y$ then $H_{\delta_{G_{z}}}=A$ (as $E_{x} \subseteq G_{z} \subseteq A$ ) and $H_{\delta_{G_{z}}} \neq F$. If $z<y$ then $G_{z}=E_{z}$ and $H_{\delta_{G}} \neq F$ again. Furthermore, $M$ does not learn $F$. Hence $H_{\delta_{F} \#^{s}}=H_{\delta_{F}}=A$ for all $s$. So $\delta_{E_{y}}$ is a semantic locking sequence for $N$ on $A$. It follows that $N$ learns $A$. This completes the verification that $N$ is a behaviourally correct learner for all the languages in its hypotheses space.

Acknowledgment. We would like to thank James Royer and Sandra Zilles for correspondence. We also thank the anonymous referees of the conference Algorithmic Learning Theory (ALT) 2007 in Sendai for comments which helped improving the paper. 


\section{References}

1. Dana Angluin. Inductive inference of formal languages from positive data. Information and Control, 45:117-135, 1980.

2. Ganesh Baliga, John Case, Wolfgang Merkle, Frank Stephan and Rolf Wiehagen. When unlearning helps. Technical Report TRA5/06, School of Computing, National University of Singapore, 2005. Conference version: G. Baliga, J. Case, W. Merkle and F. Stephan. Unlearning helps. In U. Montanari, J. D. P. Rolim and E. Welzl, editors, Proceedings of the 27th International Colloquium on Automata, Languages and Programming - ICALP 2000. Springer, Lecture Notes in Computer Science, 1853:844-855, 2000.

3. Janis Bārzdinšs. Two theorems on the limiting synthesis of functions. In Theory of Algorithms and Programs, Volume 1, Latvian State University, Riga, 210:82-88, 1974.

4. Lenore Blum and Manuel Blum. Toward a mathematical theory of inductive inference. Information and Control, 28:125-155, 1975.

5. John Case. The power of vacillation in language learning. SIAM Journal on Computing, 28:1941-1969, 1999.

6. John Case and Christopher Lynes. Inductive inference and language identification. In M. Nielsen and E. M. Schmidt, editors, Ninth International Colloquium on Automata, Languages and Programming (ICALP), Aarhus, Denmark, 1982, Proceedings. Springer, Lecture Notes in Computer Science, 140:107-115, 1982.

7. Dick de Jongh and Makoto Kanazawa. Angluin's theorem for indexed families of r.e. sets and applications. Proceedings of the Ninth Annual Conference on Computational Learning Theory, ACM Press, pages 193-204, 1996.

8. Richard Friedberg. Three theorems on recursive enumeration. Journal of Symbolic Logic, 23:309-316, 1958.

9. Mark Fulk. Prudence and other conditions on formal language learning. Information and Computation, 85:1-11, 1990.

10. E. Mark Gold. Language identification in the limit. Information and Control, 10:447-474, 1967.

11. Sanjay Jain, Daniel Osherson, James S. Royer and Arun Sharma. Systems That Learn: An Introduction to Learning Theory. MIT-Press, Boston, 1999.

12. Sanjay Jain and Arun Sharma. Prudence in vacillatory language identification. Mathematical Systems Theory, 28:267-279, 1995.

13. Sanjay Jain and Frank Stephan. Learning in Friedberg Numberings. In M. Hutter, R. Servedio and E. Takimoto, editors, Algorithmic Learning Theory: 18th International Conference, ALT 2007, Sendai, Japan, 2007, Proceedings. Springer, Lecture Notes in Artificial Intelligence, 4754:79-93, 2007.

14. Stuart Kurtz and James S. Royer. Prudence in language learning. Proceedings of the First Annual Workshop on Computational Learning Theory, MIT, Cambridge, United States, pages 143-156, 1988.

15. Steffen Lange and Thomas Zeugmann. Language learning in dependence on the space of hypotheses. Proceedings of the Sixth Annual Conference on Computational Learning Theory, Santa Cruz, California, United States, pages 127-136, 1993. 
16. Steffen Lange, Thomas Zeugmann and Shyam Kapur. Monotonic and dual monotonic language learning. Theoretical Computer Science, 155:365-410, 1996.

17. Ming Li and Paul Vitányi. An Introduction to Kolmogorov Complexity and Its Applications. Springer, Heidelberg, 1993.

18. Piergiorgio Odifreddi. Classical Recursion Theory. North-Holland, Amsterdam, 1989.

19. Daniel Osherson, Michael Stob and Scott Weinstein. Systems That Learn, An Introduction to Learning Theory for Cognitive and Computer Scientists. Bradford - The MIT Press, Cambridge, Massachusetts, 1986.

20. Emil Post. Recursively enumerable sets of positive integers and their decision problems, Bulletin of the American Mathematical Society, 50:284-316, 1944.

21. Robert Soare. Recursively Enumerable Sets and Degrees. Springer, Heidelberg, 1987.

22. Rolf Wiehagen. A thesis in inductive inference. In, J. Dix, K. Jantke and P. Schmitt, editors, Proceedings First International Workshop on Nonmonotonic and Inductive Logic, Karlsruhe, Germany, Proceedings. Springer, Lecture Notes in Artificial Intelligence, 543:184-207, 1990.

23. Thomas Zeugmann. Algorithmisches Lernen von Funktionen und Sprachen. Habilitationsschrift, Technische Hochschule Darmstadt, 1993.

24. Thomas Zeugmann and Steffen Lange. A guided tour across the boundaries of learning recursive languages. In K. P. Jantke and S. Lange, editors, Algorithmic Learning for KnowledgeBased Systems, GOSLER final report. Springer, Lecture Notes in Artificial Intelligence, 961:193-262, 1995.

25. Thomas Zeugmann, Steffen Lange and Shyam Kapur. Characterizations of monotonic and dual monotonic language learning, Information and Computation, 120:155-173, 1995.

26. Sandra Zilles. Separation of uniform learning classes. Theoretical Computer Science, 313:229265, 2004.

27. Sandra Zilles. Increasing the power of uniform inductive learners. Journal of Computer and System Sciences, 70:510-538, 2005. 